\title{
Cognitive Reactivity, Suicidal Ideation and Future Fluency: Preliminary Investigation of a Differential Activation Theory of Hopelessness/Suicidality
}

\author{
J. M. G. Williams · A. J. W. Van der Does $\cdot$ T. Barnhofer • \\ C. Crane $\cdot$ Z. S. Segal
}

Published online: 10 April 2007

(C) Springer Science+Business Media, LLC 2007

\begin{abstract}
The authors investigated whether the re-emergence of hopeless/suicidal cognitions over time can be explained within a differential activation framework. Studies $1(N=146)$ and $2(N=136)$ showed that individuals who reported suicidal ideation when depressed in the past had higher scores on the hopelessness/suicidality subscale of a measure assessing cognitive reactivity to low mood, the LEIDS. Study 3 $(N=32)$ demonstrated that self-reports on this subscale predicted changes in generativity for positive future events, an experimental measure of processes underlying hopelessness/suicidality, following sad mood induction. The results provide preliminary evidence that history of suicidal ideation is related to a specific cognitive response pattern, which may be reactivated by mild fluctuations in mood.
\end{abstract}

Keywords Depression · Suicide · Recurrence ·

Cognitive reactivity $\cdot$ Rumination $\cdot$ Hopelessness .

Future thinking $\cdot$ Vulnerability $\cdot$ Differential activation theory

\section{Introduction}

Long-term follow-up studies have shown that recovered depressed patients are at high risk for recurrences (Mueller et al., 1999). Furthermore, evidence suggests that later episodes of depression require less activation from negative life events than earlier

J. M. G. Williams $(\bowtie) \cdot$ T. Barnhofer · C. Crane

Department of Psychiatry,

Oxford University, Warneford Hospital, Warneford Lane, Oxford OX3 7JX, UK

e-mail: mark.williams@psych.ox.ac.uk

A. J. W. Van der Does

Department of Psychology, Leiden University, Leiden, Netherlands

Z. S. Segal

Department of Psychiatry, University of Toronto, Toronto, Canada 
episodes - in other words, sensitization may occur (Kendler, Thornton \& Gardner, 2000; Post, 1992; Mitchell, Parker, Gladstone, Wilhelm, \& Austin, 2003). From a cognitive science perspective, it has been suggested that sensitization (and increased risk of relapse and recurrence) is brought about by increased cognitive reactivity to small changes in depressed mood (Segal, Williams, Teasdale \& Gemar, 1996). Cognitive reactivity has been defined as the relative ease with which maladaptive cognitions or cognitive styles are triggered by mild (non-pathological) mood fluctuations (see Ingram, Miranda \& Segal, 1998; Segal \& Ingram, 1994). Such reactivity is assumed to be the observable result of an underlying differential activation process that has occurred over the learning history of the individual.

This article investigates whether the same mechanisms that underlie vulnerability to relapse and recurrence in depression in general may extend to hopelessness and suicidality in particular. Of all the symptoms of depression, hopelessness and suicidal ideation are characterized by some of the most negative global self-referent cognitions (Orbach, Mikulincer, Sirota, \& Gilboa-Schechtman, 2003). As such, hopeless and suicidal cognitions are prototypical for the kind of thinking patterns shown to be subject to differential activation. We are interested in this question because suicidal ideation is one of the most serious symptoms of depression. Epidemiological surveys suggest that around $25 \%$ of people who meet criteria for current depression in the community experience suicidal ideation as part of their depression (Goldney, Wilson, Del Grande, Fisher \& McFarlane, 2000), and the rate of suicide ideation in depressed in-patients is around 50\% (Mann, Waternaux, Haas \& Malone, 1999).

Several factors have been found to increase risk for suicidal ideation and behavior: gender (being male increases risk for completed suicide, being female increases risk for attempted suicide), lower socio-economic status, previous psychiatric disorder, the number of different psychiatric diagnoses and presence of previous suicide attempts (Kessler, Borges, \& Walters, 1999; see Joiner \& Rudd, 2000a, and Hawton \& van Heeringen, 2000 for reviews). However, knowledge of these risk factors is inadequate for predicting suicidal ideation and behavior in the individual case (Beautrais, 2004; Pokorny, 1993). Further, for the purposes of developing treatments to reduce risk of suicide, each of these factors is largely irrelevant, since they are either historical or sociodemographic, and so cannot be modified by treatment. The development of better treatment strategies is dependent upon better knowledge of the risk mechanisms; that is how these historical or demographic factors act on the individual in the present to produce suicidal reactions to current crises.

A recent study comparing formerly depressed patients with and without a history of suicidal ideation during past episodes of depression (Williams, Barnhofer, Crane \& Beck, 2005), found that previously suicidal participants showed significant decreases in interpersonal problem-solving ability following induction of sad mood. By contrast, problem-solving performance in those without a history of suicidal ideation remained relatively unchanged. These data suggest that small changes in mood may reinstate cognitive deficits that are thought to contribute to escalation of suicidal crises. This article is concerned with whether hopelessness, another established vulnerability factor which very frequently co-occurs with suicidal ideation, is subject to similar processes of reactivation.

Previous research has found that hopelessness is a critical factor in increasing the risk of suicidal behavior (Salter \& Platt, 1990; Wetzel, Margulies, Davis, \& Karam, 1980). Hopelessness is closely associated with whether individuals feel they have any reasons for living (Linehan, Goodstein, Nielsen, \& Chiles, 1983; Malone, Oquendo, Haas, Ellis, 
Li, \& Mann, 2000; Mann et al., 1999) and predicts both repetition of suicide attempts (Mann et al., 1999; Petrie, Chamberlain, \& Clarke, 1988; Scott, House, Yates, \& Harrington, 1997; Sidley, Calam, Wells, Hughes, \& Whitaker, 1999; Szanto, Reynolds, Conwell, Begley, \& Houck, 1998) and completed suicides up to 10 years later (Beck \& Steer, 1989; Beck, Brown, Berchick, Stewart, \& Steer, 1990; Fawcett et al., 1990).

However, the stability of hopelessness over time remains unclear. Although it is established that those with a history of self harm have higher hopelessness levels before and after episodes of acute psychiatric illness (Mann et al., 1999; Young, Fogg, Scheftner, Fawcett, Akiskal, \& Maser, 1996), there is evidence that hopelessness levels increase during the acute episode and diminish over the next few days (Schotte, Cools \& Payvar, 1990; Young et al., 1996). The question is whether such increases in hopelessness are simply a reaction to the negative life events that are almost always present in the recent history of those who attempt suicide (Hawton, Fagg, Simkin, Bale, \& Bond, 1997; Paykel, Prusoff, \& Myers, 1975), or whether there is an underlying factor that reflects a latent predisposition to react with hopeless cognitions to even relatively mild changes in mood. While the finding that problem-solving deteriorates following mild mood changes in formerly suicidal individuals is consistent with the idea of latent vulnerability, this might not necessarily be the case for hopelessness. Doubts are raised by a study from Young et al. (1996), who found that suicidal behavior was predicted by levels of hopelessness during remission, but not by hopelessness sensitivity, which was defined as the rate of increase from baseline levels during relapse into a full episode of depression. However, their study was focused on predicting suicide attempts by changes in hopelessness in a clinically referred sample of depressed patients. We are interested in whether hopelessness and suicidality are subject to differential activation in a less severe population with a history of previous depression with or without a history of suicidal ideation.

The differential activation model of cognitive reactivity suggests that hopelessness and suicidal ideation originally arise as features of negative thinking during early episodes of depression. During these episodes an association is formed between depressed mood and hopelessness/suicidal ideation such that future depressed mood, however caused, will activate these patterns of thinking (Joiner, 2002; Joiner \& Rudd, 2000b). In this way, hopelessness/suicidality becomes part of a 'configuration' of associations and feedback loops within the information-processing system: it enters the 'rehearsal pool' that is activated with each subsequent episode of depression. After several such episodes, the subsequent return of mood acts as the 're-instatement' of a context, similar to the processes observed in renewal of CS-CR links in animal conditioning studies (Bouton, 2002).

In summary, the differential activation model suggests that it is not the resting level of hopeless/suicidal cognitions that is important in rendering someone vulnerable to future suicidal crises. Rather, it is the ease with which these patterns of thinking can be activated that is important.

The differential activation theory of recurrence for depression has most frequently been investigated in patients once they have recovered using an experimental manipulation to 'prime' their mood. Such studies have used a variety of measures to assess the shift towards depressogenic thinking following mood induction, most commonly the Dysfunctional Attitudes Scale (DAS; Weissman, 1979), but also information processing tasks (such as tests of memory bias, dichotic listening, or the Stroop test). Although some of these studies have not replicated the finding of increased reactivity in previously depressed patients (e.g., Dykman, 1997) the balance of evidence 
finds cognitive vulnerability following priming (e.g., Ingram \& Ritter, 2000; Miranda, Gross, Persons \& Hahn, 1998; Taylor \& Ingram, 1999; Van der Does, 2002a). In two studies, high cognitive reactivity in dysfunctional attitudes, assessed following mood priming, predicted relapse and recurrence (Segal, Gemar and Williams, 1999; Segal et al., 2003; see Lau, Segal \& Williams, 2004 for review).

Recently it has been suggested that, in addition to using a mood challenge, important components of cognitive reactivity, especially the habitual pattern of responses that individuals tend to display when mood deteriorates, may be assessed by questionnaire. In two studies, the Leiden Index of Depression Sensitivity (LEIDS) was found to distinguish those who had, from those who had not experienced depression in the past (Van der Does, 2002a; Van der Does, 2005). In both these studies DAS scores were equivalent across groups.

The LEIDS follows the example of other questionnaires that assess vulnerability (for example, the Anxiety Sensitivity Index, Peterson \& Reiss, 1992), by asking conditional questions. Participants respond with how they would feel and think if they were to experience a low mood. Importantly, the LEIDS scale has not only been found to distinguish between previously depressed and never depressed people, but also to predict amount of change in Dysfunctional Attitude Scale scores following a mood challenge (Van der Does, 2002a).

To examine suicidal reactivity, Studies 1 and 2, used the revised version of the LEIDS (Van der Does, 2002a; Van der Does \& Williams, 2003), which includes a hopelessness/ suicidality subscale. This subscale measures the extent to which individuals report that they feel hopeless and have thoughts about death and suicide when they experience dysphoria. Although it is a self-report measure, it allows us to begin to examine to what extent this reactivity pattern is specific to history of suicidal ideation. The extent of such specificity can be investigated from two perspectives. First, while those who have been suicidal when last depressed may be reactive in several respects, previous suicidality should be most closely related to hopelessness/suicidal reactivity. If suicidality when depressed were found to be significantly associated with higher ratings of reactivity on all the LEIDS subscales (e.g., perfectionism), then we should conclude that previous within-episode suicidality is simply a marker of higher levels of cognitive reactivity in general. Second, the presence or absence of other symptoms that occurred during the past depressive episode, such as sleep or appetite disturbance, should not be systematically related to degree of hopelessness/suicidal reactivity. If we were to find that hopelessness/suicidal reactivity on the LEIDS was associated with the presence of all or most symptoms of depression, we should conclude that this subscale of the LEIDS was simply a measure of the extent or severity of past depressive episodes. We report analyses for both aspects of specificity. Since hopelessness/suicidal reactivity has not been examined before, we examined the data both through overall analyses of variance, looking, for example, for the interaction between presence and absence of suicidality during a past episode and the different sub-scales of reactivity, and also carrying out hypothesis-driven (pre-planned) comparisons of the effect of previous suicidality on particular sub-scales of reactivity.

Study 1 reports data from a cross-sectional study of non-depressed undergraduates, examining the association between reports of past suicidality (when depressed) and the endorsement of both hopelessness and suicidality items on the LEIDS. Study 2 examines this pattern in a community sample, adding to the first study by examining data on prior history of depression that had been obtained 6 years earlier to see if it also predicted current cognitive reactivity on LEIDS hopelessness/suicidality. 
Both Studies 1 and 2 rely on participants' self-report both for past history and for current reactivity. Study 3 therefore tests the hypothesis that, when given an experimental mood challenge, high scorers on the LEIDS hopelessness/suicidality scale would show a different pattern of activation from low scorers, using a task that does not rely on self-report. Experimental work derived from Tversky and Kahneman's (1973) availability heuristic strongly suggests that the experience of hopelessness, as with other judgments under uncertainty, is critically related to the fluency with which individuals are able to generate instances of relevant future events (see MacLeod, 1999 for review). In a series of studies MacLeod and colleagues have used a future fluency task in which participants are asked in alternating order to produce as many positive or negative events as possible that may happen to them during certain time periods in the future (e.g., week, month, year, 5-10 years). Results of these studies demonstrate that hopelessness is significantly associated with lack of fluency for positive events while there are usually no relations with fluency for negative events (MacLeod \& Byrne, 1996; MacLeod \& Cropley, 1995; MacLeod, Pankhania, Lee, \& Mitchell, 1997; MacLeod, Rose, \& Williams, 1993; O'Connor, 2003). Further, low positive future fluency is particularly pronounced in suicidal patients (e.g., MacLeod et al., 1997) Given these findings, future fluency was chosen to be the most appropriate paradigm to investigate whether LEIDS hopelessness/suicidality would predict actual reactivity in processes related to or underlying hopelessness/suicidality.

All studies were approved by the relevant Ethics Committees, and all participants gave informed consent to participate.

\section{Study 1}

The first aim of Study 1 was to test the hypothesis that suicide ideation during a past episode of depression will predict a pattern of increased hopelessness/suicidal reactivity, as assessed by the LEIDS in individuals when they are not currently depressed.

Method

\section{Participants}

Participants in this study were undergraduate (first year) psychology students. They filled in questionnaires during a mass testing session in November, which was before they had taken any course in psychopathology. Students received course credit for participating.

The questionnaires were administered with a number of other measures that were unrelated to the present research. Participants spent approximately $35 \mathrm{~min}$ filling out the questionnaires.

\section{Measures}

Depression diagnosis. The presence of current and past depression was assessed with a self-report measure, the Major Depression Questionnaire (MDQ) (Van der Does, Barnhofer, \& Williams, 2003). The measure consists of a series of questions that cover all DSM-IV diagnostic criteria for current and past major depression (American Psychiatric Association, 1994), including impact on functioning and exclusion criteria 
(e.g., mourning). Consistency of this questionnaire with diagnoses based on SCID interviews was examined in a sample of 39 individuals. All of the 19 individuals who received a diagnosis based on the interview, and 15 of the 20 individuals who did not receive a diagnosis were correctly identified by MDQ self-ratings (Sensitivity of MDQ $=100 \%$, Specificity $=75 \%$, Positive Predictive Value $=79 \% ;$ Negative Predictive Value $=100 \%$; overall kappa $=.75)$.

Depressive symptoms. The 21-item Beck Depression Inventory-2nd edition (BDI-II; Beck, Steer, \& Brown, 1996) was used to assess current (past 2 weeks) level of depressive symptoms. The Dutch translation was used (Van der Does, 2002b).

Cognitive reactivity. The Leiden Index of Depression Sensitivity (LEIDS; Van der Does, 2002a) is a self-report measure of cognitive reactivity to sad mood. The scale has been found to discriminate between never-depressed and recovered-depressed individuals (Van der Does, 2002a). Recently, a revised version has been developed that has 34 items and six subscales (Van der Does \& Williams, 2003). The names of the subscales, and sample items are: Hopelessness/Suicidality (HOP: When I feel down, I more often feel hopeless about everything; When I feel sad, I feel more that people would be better off if I were dead); Acceptance/Coping (ACC: When I am sad, I feel more like myself); Aggression (AGG: When I feel down, I lose my temper more easily); Control/ Perfectionism (CTR: When in a sad mood, I become more bothered by perfectionism); Harm Avoidance (HAV: When I feel down, I take fewer risks); Rumination (RUM: When I feel sad, I spend more time thinking about the possible causes of my moods). Internal consistencies of the subscales ranged from $\alpha=.64$ for Acceptance/Coping to $\alpha=.84$ for Hopelessness/Suicidality.

Results

\section{Participants}

The questionnaires were filled out by 146 participants. Of these, 24 reported depressive symptoms on the MDQ suggestive of a full episode and were excluded. The characteristics of the remaining sample of 122 participants are listed in Table 1. Thirty-six participants reported past symptoms fulfilling diagnostic criteria for a previous episode of depression (hereafter referred to as 'probable diagnoses' in Studies 1 and 2 to reflect the fact that diagnoses were made on the basis of questionnaire only), another six reported having had at least five symptoms over a period of less than

Table 1 Gender distribution and means and standard deviations for age and BDI scores

\begin{tabular}{llc}
\hline & \multicolumn{2}{l}{ Past depression } \\
\cline { 2 - 3 } & Yes & No \\
\hline Study 1 & & \\
$N$ (female/male) & $36(31 / 5)$ & $80(69 / 11)$ \\
Age & $19.7(3.7)$ & $19.1(3.7)$ \\
BDI-II & $11.1(9.3)$ & $6.4(5.9)$ \\
Study 2 & & \\
N (female/male) & $63(36 / 27)$ & $57(32 / 25)$ \\
Age & $46.7(7.0)$ & $49.5(6.6)$ \\
BDI-II & $8.5(5.2)$ & $5.6(5.2)$ \\
\hline
\end{tabular}


2 weeks, and 80 participants reported having never been depressed. Eighty-three percent were women, and the male/female ratio was equivalent in the never-depressed and previously depressed groups $\left(\chi^{2}(1)=.00 ; \mathrm{ns}\right)$. There was no significant difference in mean age, but groups differed significantly in their BDI-II scores, $t(114)=3.24, P<.01$. Analyses, therefore, followed up significant findings with ANCOVAs using BDI scores as a covariate.

\section{Cognitive reactivity and history of suicidality}

The first hypothesis to be examined was that individuals with a history of suicidal ideation during an episode of depressed mood would differ significantly from those with episodes of depressed mood without suicidal ideation only with respect to levels of hopelessness/suicidal reactivity on the LEIDS. For this purpose two groups of participants were created: those who reported the presence of one or both of the core symptoms of MDD for a period of at least 2 weeks and who also reported being suicidal at this time versus those who did not report being suicidal during such an episode of depressed mood. Scores on each subscale of the LEIDS were compared between these groups. Table 2 shows significance levels after co-varying out current depression levels (BDI). The Hopelessness/Suicidality subscale was markedly higher in people who had reported suicidal ideation $(P<.001)$. Scores on the other subscales of the LEIDS did not differ significantly. In other words, those who reported having been suicidal during a past episode of depressed mood reported pronounced elevation of one aspect of cognitive reactivity, hopelessness/suicidality. This finding also remained significant when differences were tested with number of symptoms (except suicidality) during the past index episode of depression entered as a covariate, $F(2,65)=5.50, P=.02$, suggesting that this effect is not due to differences in severity of previous depression. The same pattern of findings was obtained when analyses were based on sub-groups of previously and never-suicidal participants constructed to match exactly for current BDI scores ( $n=13$, BDI: $M=5.00$ in both groups), $F(1,24)=6.41, P<.05$, for Hopelessness $/$ Suicidality, all other scales $P>.10$.

Table 2 Means and Standard Deviations of LEIDS subscale and total scores by absence/presence of suicidal ideation in currently non-depressed individuals

\begin{tabular}{|c|c|c|c|c|c|c|}
\hline & \multicolumn{6}{|l|}{ LEIDS Scores } \\
\hline & HOP & $\mathrm{ACC}$ & AGG & CTR & HAV & RUM \\
\hline \multicolumn{7}{|l|}{ Study 1} \\
\hline \multicolumn{7}{|l|}{ Suicidal thoughts } \\
\hline No $(n=46)$ & $4.80(3.32)$ & $1.47(1.77)$ & $9.32(3.95)$ & $6.76(3.71)$ & 9.39 (3.68) & $10.86(4.47)$ \\
\hline Yes $(n=22)$ & $11.34(6.12)^{* * *}$ & $2.72(3.24)$ & $10.14(5.30)$ & $7.90(4.69)$ & $10.22(4.94)$ & $12.27(5.06)$ \\
\hline \multicolumn{7}{|l|}{ Study 2} \\
\hline \multicolumn{7}{|l|}{ Suicidal thoughts } \\
\hline No $(n=44)$ & $4.59(3.22)$ & $2.55(2.87)$ & $7.16(4.57)$ & $7.57(4.22)$ & $10.64(4.75)$ & $11.41(4.80)$ \\
\hline Yes $(n=51)$ & $7.59(4.93)^{* *}$ & $2.73(2.75)$ & $8.25(5.01)$ & $8.35(4.22)$ & $12.12(3.79)$ & $13.06(4.73)$ \\
\hline \multicolumn{7}{|c|}{$\begin{array}{l}\text { Note. } \mathrm{HOP}=\text { Hopelessness; } \mathrm{ACC}=\text { Acceptance/coping; } \mathrm{AGG}=\text { Aggression; } \mathrm{CTR}=\text { control } / \\
\text { perfectionism; HAV = Harm avoidance; RUM = rumination; TTL = total LEIDS score. Asterisks } \\
\text { denote significant differences in ANCOVAs with BDI scores (study } 1 \text { ) or age and BDI scores as } \\
\text { covariates (study 2) }\end{array}$} \\
\hline
\end{tabular}


In order to further investigate this difference with regard to the two components of the LEIDS hopelessness/suicidality scale, we constructed separate subscales based on the 3 items referring to suicidal thoughts and the 2 items referring to hopelessness, respectively. Internal consistencies of these subscales were $\alpha=.88$ for the suicidality component and $\alpha=.52$ for the hopelessness component; the two subscales correlated $r=.51, P<.01$. Simple ANOVAs showed that participants with and without a history of suicidal ideation differed significantly in their tendency to respond with hopelessness (previously suicidal: $M=4.45, S D=2.01$; non-suicidal: $M=3.15, S D=1.78 ; F(1,66)=7.26, P<.01)$ as well as their tendency to respond with suicidal thoughts (previously suicidal: $M=5.00$, $S D=3.71$; non-suicidal: $M=.84, S D=1.72 ; F(1,66)=39.94, P=.001)$.

A repeated measures ANOVA with $z$-transformed scores on the hopelessness subscale and the five LEIDS sub-scales assessing other aspects of reactivity as withinand group (history vs. no history of suicidal ideation) as between-subjects factor yielded a significant main effect for group, $F(1,66)=5.7, P<.05$, but no significant group by sub-scale interaction, $F(4.2,277.3)=.82, P>.10$, suggesting that the difference in hopelessness reactivity did not represent a significant deviation from the general trend of higher reactivity in the group reporting previous suicidality. However, as a further investigation of the degree of specificity of the link between prior suicidality and hopelessness reactivity, we carried out an analysis of covariance, testing the predicted difference in hopelessness reactivity with each other sub-scale covaried separately. We found that, in each case, difference on hopelessness reactivity remained significant (all $P<.05)$.

The second hypothesis to be examined was that it is only the presence of past suicidal ideation that is related to current levels of suicidal reactivity. In order to test this hypothesis, we compared suicidal reactivity in groups defined on the basis of presence or absence of each of the symptoms of depression in turn. Depressed mood and anhedonia were not used as grouping variables since selection of participants for analysis was contingent on presence of one or both of these core symptoms of depression. Table 3 shows the means (SDs) of LEIDS Hopelessness/Suicidality scores of participants in each of these groups. Of all the other non-core symptoms of depression, only 'guilt' significantly predicted suicidal reactivity. Again, significant results were followed-up with ANOVAs using BDI scores as a covariate. Results showed that presence of suicidality when depressed was associated with significantly higher score on the LEIDS Hopelessness/Suicidality scale even when BDI was entered as covariate in the model.

\section{Discussion}

The results of Study 1 are consistent with a differential activation model of hopelessness/suicidal ideation: individuals who had experienced suicidal thoughts when depressed in the past had higher scores on the LEIDS subscale Hopelessness/Suicidality than individuals who did not have these thoughts when depressed. This finding was based on both differences in reactivation of hopelessness and differences in suicidal thoughts and maintained when current level of depression (BDI) was entered as a covariate. Although differences in hopelessness reactivity did not significantly deviate from a general trend of higher reactivity in the previously suicidal group, none of the other LEIDS scales significantly discriminated between groups. The data are consistent with the hypothesis that hopeless/suicidality is triggered by mild dysphoria in individuals who have experienced suicidal thoughts when depressed in the past. 
Table 3 Means (Standard Deviations) of LEIDS Suicidality/Hopelessness scores by absence/presence of prior symptoms of depression in currently non-depressed individuals

\begin{tabular}{|c|c|c|c|c|c|c|c|}
\hline & \multicolumn{7}{|c|}{ Symptoms of Depression } \\
\hline & $\begin{array}{l}\text { Weight } \\
\text { change }\end{array}$ & Sleep & $\begin{array}{l}\text { Retardation/ } \\
\text { Agitation }\end{array}$ & Fatigue & $\begin{array}{l}\text { Concen- } \\
\text { tration }\end{array}$ & Guilt & $\begin{array}{l}\text { Suicidal } \\
\text { Ideation }\end{array}$ \\
\hline \multicolumn{8}{|c|}{ Study 1} \\
\hline \multicolumn{8}{|c|}{ No } \\
\hline$M$ & $6.62(5.2)$ & $6.4(3.9)$ & $5.63(4.9)$ & $6.00(5.3)$ & $6.55(4.5)$ & $4.96(4.4)$ & $4.80(3.3)$ \\
\hline$N$ & 46 & 35 & 23 & $11+2$ & 24 & 36 & 46 \\
\hline \multicolumn{8}{|c|}{ Yes } \\
\hline$M$ & $7.52(5.8)$ & $7.41(6.6)$ & $7.57(5.5)$ & $7.03(5.4)$ & $7.11(5.8)$ & $9.11(5.6) *$ & $11.34(6.1)^{* * *}$ \\
\hline$N$ & 22 & 33 & 45 & 57 & 44 & 32 & 22 \\
\hline \multicolumn{8}{|c|}{ Study 2} \\
\hline \multicolumn{8}{|c|}{ No } \\
\hline$M$ & $4.48(3.3)$ & $3.00(2.3)$ & $5.20(7.3)$ & $7.00(6.0)$ & $5.83(6.7)$ & $3.62(3.2)$ & $4.59(3.2)$ \\
\hline$N$ & 21 & 6 & 5 & 8 & 6 & 21 & 44 \\
\hline \multicolumn{8}{|c|}{ Yes } \\
\hline$M$ & $6.67(4.6)$ & $6.42(4.5)$ & $6.24(4.3)$ & $6.13(4.3)$ & $6.22(4.3)$ & $6.93(4.5) *$ & $7.59(4.9)^{* *}$ \\
\hline$N$ & 75 & 89 & 91 & 87 & 89 & 74 & 51 \\
\hline
\end{tabular}

Note. Asterisks denote significant differences in ANCOVAs with BDI scores (study 1) or age and BDI scores as covariates (study 2)

$* P<.05 ; * * P<.01 ; * * * P<.001$

However, this study has a number of limitations. First, the assessment of past depressive symptoms and cognitive reactivity was based on self-report with both measures administered at the same testing session. Therefore it remains possible that the participants, when asked on the LEIDS how they might react if they were in a sad mood, were simply remembering what they said on the MDQ a few minutes before. The fact that this pattern was shown on both the suicidal and hopelessness aspects of the LEIDS Hopelessness/Suicidality subscale when examined separately provides some reassurance that participants are not simply responding to questions on the same issue (past and present suicidal ideation) in the same way due to the overlap in content of the two measures. However, collecting data on past and present mental state at the same testing session remains suboptimal and does increase risk of demand effects.

Second, the study involved undergraduate students, and while they have high rates of depression and suicidality (rates comparable to the present study are reported by Cox, Enns, Borger, \& Parker, 1999; Hawton \& James 2005, and Lewinsohn, Hops, Roberts, Seeley, \& Andrews, 1993), there may be significant differences between individuals who experience a first onset of depression during late adolescence or young adulthood and those with onset of depression in later life.

\section{Study 2}

Study 2 addressed limitations of Study 1 by relating current LEIDS scores to information about history of depression collected several years prior to completion of the LEIDS in a community sample of middle-aged adults. 
Method

\section{Participants}

Participants were recruited by contacting individuals who had previously participated in a study on neuroticism and who had given their written permission to be contacted again for participation in further research. Diagnostic information on history of depression was available from answers to questions about symptoms from the DSM-IV criteria for Major Depression. Prior diagnostic status was derived using the DSM-IV algorithm except that, because it was a community-based volunteer sample, questions referred to a period of at least 4 weeks rather than 2 weeks and loss of interest was not used as an index criterion making diagnoses generally more conservative.

Of the 1,608 individuals that took part in the study 6 years previously, 419 had received a prior diagnosis of Major Depression. An attempt was made to contact a similar number of participants without depression in the past. Potential participants were contacted by letter and asked to send back a reply form to indicate whether they might be willing to take part in the research. Of the 707 subjects contacted, 224 (32\%) indicated their willingness to take part. These were sent a questionnaire booklet along with a stamped return envelope, of which a final sample of $182(81 \%)$ returned the questionnaire booklet.

\section{Measures}

Questionnaires in the booklet included the LEIDS and the BDI-II as well as several other questionnaires unrelated to the current study. Participants were instructed to fill out the questionnaire booklet at a quiet place at their home where they did not expect to be disturbed for approximately $45 \mathrm{~min}$.

\section{Results}

\section{Sample characteristics}

Of the 182 participants in the final sample, 62 (34.0\%) reported suffering from clinically significant levels of current symptoms of depression (BDI $>18)$ and were excluded from the analyses. Of the remaining sample $(N=120), 63$ participants met criteria for a prior diagnosis of Major Depression and 57 participants had never been depressed. Characteristics of the formerly depressed and never-depressed groups are shown in Table 1. Women were overrepresented amongst those who participated, comprising $56 \%$ of the sample. The male/female ratio did not differ significantly between formerly and never depressed groups, $\chi^{2}(1, N=120)=.01, P>.10$. However, the two groups differed significantly in age, $t(118)=2.2, P<.05$, and BDI scores, $t(118)=3.1, P<.001$.

\section{Suicidal reactivity and prior reports of suicidality}

Analyses concentrated on $n=95$ individuals who had reported having suffered from a period of at least 4 weeks of depressed mood in the past whether or not they fulfilled full diagnostic criteria over this time. First, individuals with and without suicidal ideation during this prior episode were compared with regard to LEIDS reactivity scores. Again, after entering age and BDI as covariates, LEIDS Hopelessness/Suicidality scores differed significantly between these two groups, $F(3,91)=8.37, P<.01$. This finding 
also remained significant when number of symptoms during the past index episode of depression was entered as a covariate, $F(2,93)=7.92, P<.01$, thus suggesting that differences were not due to severity of past depression. None of the other LEIDS scales yielded significant differences. The same pattern of findings emerged when differences were tested between groups of previously and never-suicidal participants that were exactly matched for their BDI scores ( $n=30$, BDI: $M=6.60$ in each group), $F(1$, $58)=10.05$, for Hopelessness/Suicidality, all other scales $P>.10$.

Separate analyses of the hopelessness and suicidality components of the Hopelessness/Suicidality scale showed that participants with and without a reported history of suicidality differed both in their tendency to respond with hopelessness (previously suicidal: $M=4.42, S D=2.09$; non-suicidal: $M=3.43, S D=2.07 ; F(1,94)=5.40$, $P=.02$ ) and their tendency to respond with suicidal thoughts (previously suicidal: $M=3.25, S D=3.27$; non-suicidal: $M=1.16, S D=1.82 ; F(1,94)=14.20, P=.001)$. Internal consistencies of the subscales were $\alpha=.74$ for the hopelessness component and $\alpha=.82$ for the suicidality component; the two subscales correlated $r=.59, P<.01$.

A repeated measures ANOVA with z-transformed scores on the hopelessness subscale and the five LEIDS scales assessing other aspects of reactivity as within- and group (history vs. no history of suicidal ideation) as between-subjects factor did not yield a significant interaction between LEIDS scores and group, $F(3.7,351.3)=.79$, $P=.52$, indicating that the size of the group difference in hopelessness reactivity did not deviate significantly from the general trend of higher reactivity in those with previous suicidal ideation, $F(1,93)=3.36, P=.07$. However, an analysis of covariance showed that group differences in hopelessness reactivity remained significant when tested with Acceptance/Coping, Aggression and Control/Perfectionism entered as covariates, all $P<.05$, and were at trend level when scores on Harm Avoidance, $F(1,92)=2.68$, $P=.10$, and Rumination, $F(1,92)=2.58, P=.11$, were entered as covariates.

Second, LEIDS Hopelessness/Suicidality scores were compared between groups defined on the basis of presence or absence of non-core symptoms (see Table 3). The pattern of results replicated Study 1. Of the other non-core symptoms of depression, only presence or absence of worthlessness/guilt was associated with suicidal reactivity, $F$ $(3,91)=6.11, P<05$.

\section{Discussion}

Study 2 replicated the findings of the first study and showed that similar results are obtained when self-reports of hopelessness/suicidal cognitive reactivity are related to history of depression assessed several years prior to completion of the LEIDS. The results also indicate that the relation between past history of suicidal ideation and current ratings of hopelessness/suicidal reactivity is generalizable to an older community sample of volunteers.

The findings of studies 1 and 2 suggest that individuals with a history of suicidal ideation during episodes of sad mood report higher levels of hopelessness/suicidal reactivity during times of recovery. Is suicidal ideation when depressed in the past simply a marker of higher levels of cognitive reactivity in general? While group differences in hopelessness reactivity did not deviate sufficiently from other aspects of reactivity to yield a significant group by sub-scale interaction (the strongest form of the specificity hypothesis), nevertheless it was only the hopelessness/suicidality subscale of the LEIDS which yielded significant differences between groups. Therefore although the results do not support the strongest form of specificity, the results of our planned 
comparisons are consistent with a weaker form of specificity: that suicidality during past episodes was significantly linked with rating of continued reactivity in the hopelessness/ suicidality domain, and this predicted difference largely remained when other aspects of reactivity were entered as covariates.

Furthermore, in both studies, hopelessness/suicidality reactivity was significantly related to prior suicidal ideation, and to a lesser extent, with worthlessness/guilt. It was not associated with other symptoms of depression.

The overall pattern of these findings is consistent with a differential activation model of hopelessness/suicidality. However, interpretation of the results relies heavily on the validity of the LEIDS as a measure of reactivity and the more general question of whether it is possible to assess relevant aspects of cognitive reactivity by self-report. An underlying assumption of cognitive reactivity models is that mood disturbance activates latent vulnerability processes which would not be observable under neutral conditions. From this perspective the validity of asking individuals to report on their own patterns of cognitive reactivity when euthymic is questionable. However the rationale of the LEIDS is based on the assumption that patterns of reactivity, although mood dependent, may become habitual in nature and thus that individuals may be able to reflect and report upon their patterns of reactivity to sad mood as a repeatedly appearing and characteristic response.

Although the results of Study 2 suggest that our findings are not due to a general reporting bias at time of testing, it may still be argued that self-descriptions on the LEIDS reflect memory for symptoms during prior episodes of depression rather than patterns of reactivity as a habitual response to small changes in mood. A crucial question therefore is whether LEIDS Hopelessness/Suicidality scores predict actual reactivation of behavioral markers of hopelessness/suicidality at a later time. Experimental tests of this assumption would involve a direct measure of processes related to the experience of hopelessness/ suicidality, using a measure that was distinct from the LEIDS in both content and method. A series of studies by MacLeod and colleagues has shown that an important mechanism underlying hopelessness and pronounced in parasuicide patients is the inability to generate positive events that might occur in the future (MacLeod \& Byrne, 1996; MacLeod \& Cropley, 1995; MacLeod et al., 1997; MacLeod et al., 1993; O'Connor, 2003).

In order to investigate this question a subgroup of participants from Study 2 participated in a further experimental study in which this behavioral fluency measure was assessed before and after a negative mood induction. Based on the above findings, we hypothesized that high scorers on the LEIDS Hopelessness/Suicidality subscale would have greater difficulty in generating positive future events following a mood challenge. Furthermore, as the LEIDS is designed as a measure of reactivity, correlations with baseline levels of future fluency were expected to be low and non-significant. Finally, we predicted no relations with change in fluency for negative events, since this is generally found not to be associated with hopelessness (MacLeod et al., 1993; O'Connor, 2003).

\section{Study 3 \\ Method \\ Participants}

The sample consisted of a subgroup of 40 participants from the local area who had participated in Study 2 and who had agreed additionally to take part in an experimental 
study on cognitive reactivity. Of the total sample, 8 participants had to be excluded from analyses because of a current diagnosis of Major Depression $(n=4)$ or current suicidality $(n=4)$ as assessed by clinical interview (see below). The remaining sample $(N=32)$ consisted of 19 women and 13 men with an average age of $M=49.3(S D=6.6)$ years. Current and prior diagnostic status was assessed by trained post-doctoral psychologists using the Structured Clinical Interview for DSM-IV (SCID, First, Spitzer, Gibbon, \& Williams, 1996). Thirteen (7 female/6 male) of the participants had experienced an episode of depression without suicidal ideation, 5 (4 female/1 male) had experienced an episode of depression with suicidal ideation and 14 ( 8 female/6 male) participants had never been depressed. Current BDI scores in previously depressed subjects with suicidal ideation $(M=13.6, S D=8.7)$ and previously depressed subjects without suicidal ideation $(M=10.1, S D=5.7)$ were significantly higher than in participants without a lifetime diagnosis of depression $(M=5.6, \quad S D=7.1 ; t$ $(30)=2.2, P=.03)$. One participant had attempted suicide in the past. In terms of previous and current treatment, ten had previous treatment with antidepressant medication, and four were currently taking such medication. Two had been hospitalized in the past, nine had previous psychotherapy or counseling, two had received cognitive behavior therapy and two were currently in psychotherapy.

In terms of lifetime co-morbid diagnoses, one had prior alcohol dependence, and one non-alcohol substance dependence; four had a history of panic disorder, three agoraphobia, three social phobia, four specific phobia, two obsessive-compulsive disorder, two General Anxiety Disorder, one anorexia, one bulimia. There were no participants with a history of psychosis, nor of Post Traumatic Stress Disorder, nor of Borderline Personality Disorder.

\section{Materials}

Future Fluency Task. Future fluency was assessed before and after a mood induction. Following the procedure described by MacLeod et al. (1997) participants were given four time intervals (next week, next month, next year and next 5-10 years) and were asked in alternating order to come up with as many events as they could think of, either that they would look forward to happening (positive future events) or that they would not look forward to happening (negative future events). All four time intervals were presented once before and once after the mood induction with participants randomly allocated to receive positive event instructions for two of the time intervals and negative event instructions for the other two time intervals. For each time interval, participants were given $30 \mathrm{~s}$.

Mood induction. In order to induce negative mood a combined mood induction using sad music and Velten statements (Clark \& Teasdale, 1985) was administered. Participants listened to Prokofiev's "Russia under the Mongolian Yoke" (Alexander Nevsky Suite) played at half speed over a period of 8 min while reading a set of 32 negative Velten statements (e.g., "Everything seems futile, pointless" or "I get no satisfaction from the things I do"). Mood was measured at the beginning and end of the mood induction and the experiment using two VAS scales on which participants indicated how "despondent" and "happy" they felt at the moment.

\section{Procedure}

Participants had filled out the LEIDS on average 1-2 weeks prior to their appointment for the experimental study. Testing started with the structured clinical interview. Future 
Fluency was assessed before and immediately after the mood induction. Following the experimental session, participants filled out several diagnostic questionnaires including the Beck Depression Inventory.

Results

\section{Mood Induction}

In order to check effectiveness of the mood induction procedure, VAS scores for despondency and happiness were subjected to separate repeated-measures ANOVAs with time (before experiment, before mood induction, after mood induction, after experiment) as the single within-subjects factor. Both of these analyses yielded significant main effects (despondency: $F(3,93)=33.1, P<.01$; happiness: $F$ $(3,93)=31.3, P<.01)$. Despondency and happiness changed significantly from the beginning to the end of the mood induction (despondency: before mood induction $M=15.0, \quad S D=17.5$, after mood induction: $M=35.6, \quad S D=24.1, \quad M D=-20.5$, $\mathrm{SE}=2.8, P<.01$; happiness: before $\operatorname{mood}$ induction $M=67.0, S D=16.3$, after mood induction: $M=44.9, S D=17.1, M D=22.1, \mathrm{SE}=2.5, P<.01$ ) with the significant differences from baseline levels on both scales being retained until the end of the experimental session (despondency: end of experiment: $M=34.1, \quad S D=27.1$, $M D=-19.0, \mathrm{SE}=3.6, P<.01$; happiness: end of experiment: $M=49.2, S D=24.3$, $M D=17.7, \mathrm{SE}=3.7, P<01)$. Mood change from the beginning to the end of the induction was not significantly related to scores on the LEIDS Hopelessness/Suicidality scale ( $\Delta$ happiness: $r=.04, p=.82 ; \Delta$ despondency: $r=-.16, P=.37$ ).

\section{LEIDS Hopelessness/Suicidality and Future Fluency}

Participants produced an average of $M=7.7(S D=2.1)$ positive and $M=5.3$ $(S D=2.1)$ negative events before and $M=5.7(S D=2.0)$ positive and $M=4.6$ $(S D=2.0)$ negative events after the mood induction. The average LEIDS Hopelessness/ Suicidality score was $M=5.4(S D=4.5)$. Correlations between LEIDS Hopelessness/ Suicidality scores and future fluency for positive and negative events are shown in Table 4. Consistent with the hypotheses, higher LEIDS Hopelessness/Suicidality scores were associated with greater decreases in positive future fluency from pre to post mood induction and lower fluency for positive events following mood induction. The correlation between LEIDS Hopelessness/Suicidality and negative future fluency approached significance for fluency before the mood induction and was significant for fluency after the mood induction, but there was no relation between change in negative future fluency and LEIDS scores. Analyses based on subscales of items referring to hopelessness and suicidality, respectively, showed that correlations were mainly carried by the hopelessness facet of the scale, which was significantly related to change in positive future fluency (see Fig. 1). In contrast, the correlation between change in positive future fluency and the suicidality subscale, though in the same direction, did not reach significance (see Table 4).

In order to take account of baseline levels of future fluency and current symptoms of depression, change in positive future fluency was predicted in a hierarchical regression analysis where future fluency (pre mood induction) and BDI scores were entered into the equation in the first and LEIDS Hopelessness/Suicidality in the second step. At step 1 baseline levels of positive future fluency proved to be a significant predictor of change 
Table 4 Intercorrelations Between LEIDS Hopelessness/Suicidality and Future Fluency Scores Pre and Post Mood Induction $(N=32)$

\begin{tabular}{|c|c|c|c|c|c|c|}
\hline & \multicolumn{3}{|c|}{ Positive future fluency } & \multicolumn{3}{|c|}{ Negative future fluency } \\
\hline & Pre & Post & Pre-post & Pre & Post & Pre-post \\
\hline LEIDS Hop/Suic ${ }^{\mathrm{a}}$ & -.01 & $-.51 * *$ & $.40 * \mathrm{~b}$ & .34 & $.39 *$ & -.03 \\
\hline Hop & -.05 & $-.64 * *$ & $.48 * *$ & .35 & .34 & .02 \\
\hline Suic & .01 & -.31 & .26 & .28 & $.37 *$ & -.08 \\
\hline
\end{tabular}

Note. ${ }^{\mathrm{a}}$ LEIDS Hop/Suic $=$ LEIDS Hopelessness/Suicidality, Hop = Hopelessness items only, Suic $=$ Suicidality items only

$\mathrm{b}$ Positive correlation indicates larger reduction (pre minus post) in fluency for higher scores on LEIDS Hop/Suic

$* P<.05 ; * * P<.01$

while this was not the case for scores on the BDI. LEIDS Hopelessness/Suicidality predicted changes in positive future fluency at step 2 over and above the influence of baseline levels and BDI scores. A summary of the hierarchical regression analysis is given in Table 5. When similar regression analyses were run for the two facets of the scale, the hopelessness subscale emerged as a significant predictor, $\beta=.49, P=.001$, $\Delta R^{2}=.19$, while this was not the case for the suicidality subscale, $\beta=.21, P=.13$, $\Delta R^{2}=.04$.

Finally, in order to examine the effect of co-morbid diagnoses, we computed a total co-morbity index by summing the number of past diagnoses for each participant. Twelve participants had a history of one co-morbid diagnosis, two individuals had two such diagnoses, one had three and one had six. Examining the correlation of this prior history on reactivity showed that total co-morbidity was marginally associated with LEIDShopelessness $(r=.31, P<.10 ; r h o=.36, P=.04)$, but not with LEIDS-suicidality $(r=-.05 ; r h o=.14)$. Entering co-morbidity into a partial correlation analysis of

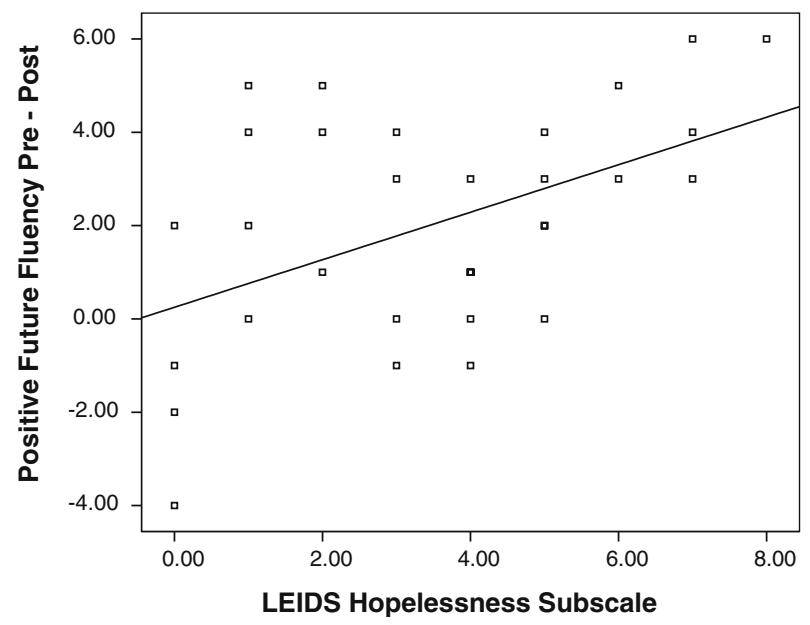

Fig. 1 Scatter-plot showing association between hopelessness reactivity on the LEIDS and change in fluency of positive events following mood challenge (note, higher pre-post change scores reflect greater deterioration in fluency performance). 
Table 5 Summary of hierarchical regression analysis for variables predicting change in future fluency for positive events $(N=32)$

\begin{tabular}{llll}
\hline Variable & $B$ & SE $B$ & $\beta$ \\
\hline Step 1 & & & $.67^{* * *}$ \\
Pos future fluency Pre & .10 & .17 & .28 \\
BDI & .79 & .05 & $.65^{* *}$ \\
Step 2 & & & .15 \\
Pos future fluency Pre & .76 & .15 & $.36^{*}$ \\
BDI & .05 & .05 & .07 \\
LEIDS Hop/Suic & .19 & . & \\
\hline
\end{tabular}

Note. $R^{2}=.45$ for step $1 ;\left(R^{2}=.12\right.$ for step $2(P \mathrm{~s}<.05)$. Pos Future Fluency Pre $=$ Number of positive future events generated before the mood induction, BDI = Beck Depression Inventory, LEIDS Hop/ Suic $=$ LEIDS Hopelessness/Suicidality. Change in Future Fluency for Positive Events was computed as fluency pre induction fluency minus post induction fluency, thus, leading to higher scores for higher decreases in fluency

$* P<.05 ; * * P<.01$

LEIDS-hopelessness with change in positive future fluency made no difference to the correlation $(r=-.59 ; P<.001)$.

\section{Discussion}

The results of Study 3 support the prediction that self-reports on the LEIDS hopelessness/suicidality scale reflect individual tendencies to respond to small changes in mood with increases in those processes known to underlie the experience of hopelessness. Consistent with the concept of the LEIDS as a measure of cognitive reactivity, hopelessness/suicidality scores predicted change in positive future fluency following mood induction over and above the influence of current symptomatology while being unrelated to baseline levels of positive future fluency. This association was stronger for the hopelessness aspect of the hopelessness/suicidality subscale. However there was no significant difference between the magnitude of the correlation coefficients between the hopelessness and suicidal reactivity items and positive future fluency change, suggesting a similar relationship holds in both cases.

\section{General Discussion}

The present studies were designed as a preliminary test of a differential activation model of hopelessness/suicidality. This model assumes that during previous episodes of depression, an association is formed between depressed mood and hopeless and suicidal cognitions establishing a response pattern that may easily be reinstated when mood deteriorates at later times, thus increasing risk for further suicidal crises.

We investigated whether non-depressed individuals who had experienced suicidal ideation during a past episode of depressed mood, would tend to report being more likely to experience hopelessness and suicidal thoughts during dysphoric mood states than individuals who had also been depressed in the past but had not experienced suicidal ideation. The results of two separate studies supported this association: history of suicidal ideation was significantly related to tendencies to respond to mild changes in mood with a pattern of thinking related to suicidality. The fact that this reactivity was shown not only in explicitly suicidal thoughts (which might have arisen through a simple 
confounding of predictor and criterion variables) but also on thoughts of hopelessness, provides some reassurance that one is seeing evidence of vulnerability to react with a relatively distinctive pattern of thinking. These relationships were found in college students who retrospectively rated their past depressive symptoms and in an older community-based volunteer sample from whom a depression history (including suicidal ideation) had been obtained 6 years earlier. Findings in Study 1 and 2 were exclusively based on self-report. The validity of these self-reports was tested in Study 3. LEIDS Hopelessness/Suicidality scores, particularly its hopelessness aspect, predicted actual mood-related changes in a behavioral measure underlying hopelessness and impaired in suicidal patients, positive future fluency, while being unrelated to baseline levels of fluency.

The findings of studies 1 and 2 contrast with the results by Young et al. (1996), who found that hopelessness sensitivity, measured as the rate of increase of self-rated hopelessness from baseline as a function of depression severity during relapse into a full episode, was unrelated to later suicide attempts. Interpretation of this seeming inconsistency is difficult, as it remains unclear how rate of increase over a period of depression relates to measures of cognitive reactivity used in our study. Unlike the study by Young et al., our research did not include prospective assessments of suicidal ideation and, while findings from Study 3 established validity of LEIDS self-reports with regard to hopelessness reactivity, no comparable evidence was gathered for selfreports of suicidal reactivity, leaving implications with regard to later occurrences of suicidal ideation more open. In the study by Young et al., associations between suicide attempts and rates of increase may have been obscured if, as suggested by relations between baseline hopelessness and suicide attempts, some of the patients at highest risk suffered from chronically elevated levels of hopelessness even during remission, thus, restricting their range of increase during full episodes. Furthermore, in a full episode, self reports of hopelessness will increase in many patients due to a range of external circumstances in addition to cognitive reactivity. The advantage of experimental induction of mood in the laboratory is the possibility of observing subtle changes that can more reliably be attributed to changes in mood, and cannot be due to changes in life circumstances.

In interpreting our findings, a number of limitations have to be borne in mind. First, the sample sizes were relatively small, so we must be cautious about generalizing from these data. Response rates were also relatively low in studies 1 and 2 and the absence of a gender difference in rates of depression suggests that these findings will require replication in more representative samples.

Second, the hypothesis that there is specificity in patterns of hopeless/suicidal reactivity amongst previously suicidal individuals should be regarded with caution at this stage. The pattern of data suggested that the relation between history of suicidality and hopelessness reactivity showed some signs of specificity, both in the sense that other depressive symptoms, with the exception of guilt, did not predict hopelessness reactivity, and in the sense that other aspects of the LEIDS were not significantly predicted by a history of suicidal ideation. Further these findings remained when previously suicidal and non-suicidal groups were exactly matched in terms of current BDI and after controlling for the severity of past depressive episodes. However it is possible that the study lacked the power to detect differences on the other LEIDS subscales that might actually exist. Additionally when a more stringent definition of specificity was tested-comparing the magnitude of group differences in hopelessness reactivity and other aspects of cognitive reactivity—both studies failed to 
produce a significant interaction of group and scale. This indicates that the magnitude of group differences in hopelessness reactivity did not deviate significantly from the overall pattern of differences on the other LEIDS scales, mainly due to general trends towards higher reactivity in the previously suicidal group. Altogether, interpretation of these results would suggest that, in individuals with previous suicidality, pronounced increases in hopelessness reactivity are likely to be accompanied by unsystematic increases in other aspects of reactivity, particularly ruminative and avoidant tendencies, both of which reduced differences in hopelessness reactivity to near trend levels when entered as covariates in Study 2 (though not Study 1). Larger samples are needed to provide stronger tests of the uniqueness of suicidal ideation as a specific predictor of hopelessness/suicidal reactivity and the hypothesis of specificity in patterns of hopelessness/suicidal cognitive reactivity should, for the time being, be interpreted with caution.

Tests of the relation between hopelessness reactivity and different symptoms of depression revealed significant findings in both studies not only for suicidality but also for guilt. This relation with guilt was unexpected and interpretation must, therefore, remain speculative. It is interesting to note, however, that recent attempts at identifying dimensional structures of depressive symptoms have highlighted the relation between guilt and suicidality (Korszun et al., 2004). From a cognitive perspective, guilt is likely to be an indicator of negative self-attributions that may be similar in nature to beliefs underlying hopelessness. Both, guilt about the past and hopelessness about the future, may contribute to a sense of entrapment that may ultimately fuel suicidal ideation.

A third set of limitations relates to the fact that the LEIDS primarily deals with the experience of suicidal ideation and feelings of hopelessness, rather than with reactivation of suicidal behavior. Consistent with this, the vast majority of previously suicidal participants in these studies had not made a suicide attempt. Clearly differences may exist between suicide ideators and suicide attempters with regard both to the degree and the consequences of suicidal cognitive reactivity (Rudd, Joiner, \& Rajab, 1996). A number of factors are likely to moderate the relationship between the ease of activation of suicidal thoughts in response to relatively mild states of low mood and the development of sustained suicidal ideation or subsequent suicidal behavior. Further studies, utilising clinical populations with higher base rates of attempted suicide, will be required in order to examine such interactions in more detail.

Whilst studies 1 and 2 examined participants differing in their history of suicidal ideation, Study 3 compared the effects of self-reported hopelessness/suicidal cognitive reactivity on a behavioral measure closely associated with hopelessness. While Study 3 therefore provides useful validation of the LEIDS, the next step in examining hopelessness/suicidal cognitive reactivity would be to explore whether individuals with a history of suicidal behavior would display more pronounced deteriorations in positive future fluency following mood induction, an analysis precluded in the current study by the relatively small number of previously suicidal participants.

Finally, the data were collected in undergraduate and community samples rather than patients referred for treatment, so we did not have co-morbidity data for participants in Studies 1 and 2. It is possible that some participants in these studies had past or current diagnoses other than depression. On the other hand, there is a great deal of evidence that, even in other psychiatric conditions, it is depression and hopelessness that is the critical mediator for serious suicidal ideation and behavior (Beautrais et al., 1996; Mann et al., 1999). The findings of Study 3 were consistent with this evidence. In this study, 
co-morbidity data was available, but entering it into a partial correlation analysis made no difference to the prediction of change in positive future fluency by LEIDS hopelessness following the mood induction.

The present research has a number of implications. First, hopelessness and suicidal ideation are often regarded as symptoms of severe depression. The present study indicates, however, that if they have co-occurred in the past, suicidal thoughts and their precursors such as hopelessness may be triggered by mild mood fluctuations, and thus can also be part of a mild or sub-clinical episode of depression. This process may be involved in the escalation from mild dysphoria to severe negative moods and suicidal ideation. Risk of further suicidal ideation and behavior, therefore, is determined by the ease with which these reverberatory patterns of processing become re-established, following recovery, in the face of mild negative affect.

Second, these findings may help us understand the apparent divergence between previous treatment studies' proven efficacy in treating important continuous variables (hopelessness, depression and problem solving) and their relatively weak effects on suicidal behavior itself (Hawton et al., 1998). It suggests that the critical risk factor is not the resting level of these variables assessed after treatment and during follow-up or when patients are in remission, but rather how easily activated these variables are in response to mood challenge. Such activation vulnerability has not been assessed in any treatment study to date.

Finally, if future suicidal behavior is determined in part by the ease with which hopelessness and future fluency deficits are activated by mood change, then therapists need to be cautious in drawing conclusions from apparent improvements in baseline levels of hopelessness in their patients. Focusing on reducing hopelessness itself is not sufficient, nor does a lower level of hopelessness at the end of treatment provide reassurance that the underlying vulnerability has been treated. This is of particular concern following antidepressant treatment for depression. Antidepressants are effective in reducing depression levels and reducing levels of dysfunctional attitudes as assessed when euthymic. However Segal et al. $(1999,2006)$ have demonstrated that these patients remain susceptible to mood-activated cognitive reactivity, showing increased dysfunctional attitudes following mood induction. There is the danger that clinicians will see a patient's reduction in hopelessness as indicating treatment success, but fail to see that the underlying cognitive reactivity has not changed. This will be especially problematic if the clinician decides to reduce the dose of medication.

Recent advances in Cognitive Therapy for suicidal behavior (Brown et al., 2005) include a reactivity procedure towards the end of therapy to investigate what pattern of thinking and impulses come to mind under such 'activated' conditions. Although to date this component has not been formally evaluated our data strongly supports the wisdom of such methods. The present series of studies shows that hopelessness and suicidality may disappear altogether when patients are euthymic, but remain ready to be reactivated when depression returns. It is this vulnerability towards which our therapeutic efforts should be focused.

Acknowledgements The authors wish to thank Danielle Duggan, Melanie Fennell, Silvia Garnsey and Wendy Swift for help with this research. This research was supported by the Wellcome Trust GR067797 


\section{References}

American Psychiatric Association (1994). Diagnostic and statistical manual of mental disorders (4th Ed) (DSM-IV). Washington, DC: Author.

Beautrais, A. L. (2004). Further suicidal behavior among medically serious suicide attempters. Suicide and Life-Threatening Behavior, 34, 1-11.

Beautrais, A. L., Joyce, P. R., Mulder, R. T., Fergusson, D. M., Deavoll, B. J., \& Nightingale, S. K. (1996). Prevalence and co-morbidity of mental disorders in persons making serious suicide attempts: A case-control study. American Journal of Psychiatry, 153, 1009-1014.

Beck, A. T., Brown, G., Berchick, R. J., Stewart, B. L., \& Steer, R. A. (1990). Relationship between hopelessness and ultimate suicide-A replication with psychiatric outpatients. American Journal of Psychiatry, 147, 190-195.

Beck, A. T., \& Steer, R. A. (1989). Clinical predictors of eventual suicide: A 5- to 10-year prospective study of suicide attempters. Journal of Affective Disorders, 17, 203-209.

Beck, A. T., Steer, R. A., \& Brown, G. K. (1996). The Beck Depression Inventory-2nd edition (BDI-II). San Antonio, TX: The Psychological Corporation.

Bouton, M. E. (2002). Context, ambiguity, and unlearning: Sources of relapse after behavioral extinction. Biological Psychiatry, 52, 976-986.

Brown, G. K., Ten Have, T., Henriques, G. R., Xie, S. X., Hollander, J. E., \& Beck, A. T. (2005). Cognitive therapy for the prevention of suicide attempts: A randomized controlled trial. JAMA, 294, $563-570$

Clark, D. M., \& Teasdale, J. D. (1985). Constraints on the effects of mood on memory. Journal of Personality and Social Psychology, 48, 1595-1608.

Cox, B. J., Enns, M. W., Borger, S. C., \& Parker, J. D. A (1999) The nature of the depressive experience in analogue and clinically depressed samples. Behaviour Research and Therapy, 37, 15-24.

Dykman, B. M. (1997). A test of whether negative emotional priming facilitates access to latent dysfunctional attitudes. Cognition \& Emotion, 11, 197-222.

Fawcett, J., Scheftner, W. A., Fogg, L., Clark, D. C., Young, M. A., Hedeker, D. et al. (1990). Timerelated predictors of suicide in Major Affective-Disorder. American Journal of Psychiatry, 147, 1189-1194.

First, M. B., Spitzer, R. L., Gibbon, M., \& Williams, J. B. (1996). User's guide for the Structured Clinical Interview for DSM-IV Axis I Disorders. Washington, DC: American Psychiatric Press.

Goldney, R. D., Wilson, D., Del Grande, E., Fisher, L. J., McFarlane, A. C. (2000). Suicidal ideation in a random community sample: Attributable risk due to depression and psychosocial and traumatic events. Australian and New Zealand Journal of Psychiatry, 34, 98-106.

Hawton, K., Arensman, E., Townsend, E., Bremner, S., Feldman, E., Goldney, R., Gunnell, D., Hazell, P., van Heeringen, C., House, A., Owens, D., Sakinowsky, I., \& Traskman-Bendz, L. (1998). Deliberate self-harm: A systematic review of the efficacy of psychosocial and pharmacological treatments in preventing depression. British Medical Journal, 317, 441-447.

Hawton, K., Fagg, J., Simkin, S., Bale, E., \& Bond, A. (1997). Trends in deliberate self-harm in Oxford, 1985-1995-Implications for clinical services and the prevention of suicide. British Journal of Psychiatry, 171, 556-560.

Hawton, K., \& James A. 2005 Suicide and deliberate self harm in young people. British Medical Journal, $330,891-894$.

Hawton, K., \& van Heeringen, K. (Eds.) (2000). The international handbook of suicide and attempted suicide. New York, NY: John Wiley.

Ingram, R. E., Miranda, J., \& Segal, Z. V. (1998) Cognitive vulnerability to depression. New York: Guilford Press.

Ingram, R. E., \& Ritter, J. (2000). Vulnerability to depression: Cognitive reactivity and parental bonding in high-risk individuals. Journal of Abnormal Psychology, 109, 588-596.

Joiner, T. E. (2002). The trajectory of suicidal behavior over time. Suicide and Life-Threatening Behavior, 32, 33-41.

Joiner, T. E., \& Rudd, M. D. (Eds.) (2000a). Suicide science: Expanding the boundaries. New York, NY: Kluwer Academic/Plenum Publishers.

Joiner, T. E., \& Rudd, M. D. (2000b) Intensity and duration of suicidal crises vary as a function of previous suicide attempts and negative life events. Journal of Consulting and Clinical Psychology, 68, 909-916.

Kendler K. S., Thornton L. M., Gardner C. O. (2000). Stressful life events and previous episodes in the etiology of major depression in women: An evaluation of the 'kindling' hypothesis. American Journal of Psychiatry, 157, 1243-1251. 
Kessler, R. C., Borges, G., \& Walters, E. E. (1999). Prevalence of and risk factors for lifetime suicide attempts in the national comorbidity survey. Archives of General Psychiatry, 56, 617-626.

Korszun, A., Moskvina, V., Brewster, S., Craddock, N., Ferrero, F., Gill, M. et al. (2004). Familiality of symptom dimensions in depression. Archives of General Psychiatry, 61, 468-474.

Lau, M. A., Segal, S. Z., \& Williams, J. M. G. (2004). Teasdale's differential activation hypothesis: Implications for mechanisms of depressive relapse and suicidal behaviour. Behaviour Research and Therapy, 42, 1001-1004.

Lewinsohn, P. M., Hops, H., Roberts, R. E., Seeley, J. R., \& Andrews, J. A. (1993) Adolescent psychopathology: I. Prevalence and incidence of depression and other DSM-III-R disorders in high school students. Journal of Abnormal Psychology, 102, 133-144.

Linehan, M. M., Goodstein, J. L., Nielsen, S. L., \& Chiles, J. A. (1983). Reasons for staying alive when you are thinking of killing yourself. Journal of Consulting and Clinical Psychology, 51, 276-286.

MacLeod, A. K. (1999). Prospective cognition. In T. Dalgleish, \& M. J. Power (Eds.), Handbook of cognition and emotion. New York, NY: John Wiley \& Sons Ltd.

MacLeod, A. K., \& Byrne, A. (1996). Anxiety, depression, and the anticipation of future positive and negative experiences. Journal of Abnormal Psychology, 105, 286-289.

MacLeod, A. K., \& Cropley, M. L. (1995). Depressive future-thinking-the role of valence and specificity. Cognitive Therapy and Research, 19, 35-50.

MacLeod, A. K., Pankhania, B., Lee, M., \& Mitchell, D. (1997). Parasuicide, depression and the anticipation of positive and negative future experiences. Psychological Medicine, 27, 973-977.

MacLeod, A. K., Rose, G. S., \& Williams, J. M. G. (1993). Components of hopelessness about the future in parasuicide. Cognitive Therapy \& Research, 17, 441-455.

Malone, K. M., Oquendo, M. A., Haas, G. L., Ellis, S. P., Li, S. H., \& Mann, J. J. (2000). Protective factors against suicidal acts in major depression: Reasons for living. American Journal of Psychiatry, 157, 1084-1088.

Mann, J. J., Waternaux, C., Haas, G. L., \& Malone, K. M. (1999) Towards a clinical model of suicidal behavior in psychiatric patients. American Journal of Psychiatry, 156, 181-189.

Miranda, J., Gross, J. J., Persons, J. B., \& Hahn, J. (1998). Mood matters: Negative mood induction activates dysfunctional attitudes in women vulnerable to depression. Cognitive Therapy and Research, 22, 363-376.

Mitchell, P. B., Parker, G. B., Gladstone, G. L., Wilhelm, K., \& Austin, M. P. V. (2003). Severity of stressful life events in first and subsequent episodes of depression: The relevance of depressive subtype. Journal of Affective Disorders, 73, 245-252.

Mueller, T. I., Leon, A. C., Keller, M. B., Solomon, D. A., Endicott, J., Coryell, W., Warshaw, M., \& Maser, J. D. (1999). Recurrence after recovery from major depressive disorder during 15 years of observational follow-up. American Journal of Psychiatry, 156, 1000-1006.

O'Connor, R. C. (2003). Suicidal behaviour as a cry of pain: Test of a psychological model. Archives of Suicide Research, 7, 1-12.

Orbach, I., Mikulincer, M., Sirota, P., \& Gilboa-Schechtman, E. (2003) Mental Pain: a multidimensiopanl operationalization and definition. Suicide and Life-Threatening Behavior, 33, 219-230.

Paykel, E. S., Prusoff, B. A., \& Myers, J. K. (1975). Suicide attempts and recent life events: A controlled comparison. Archives of General Psychiatry, 32, 327-333.

Peterson, R. A., \& Reiss, S. (1992). Anxiety Sensitivity Index Manual, 2 nd edn. Worthington, OH: IDS Publishing Corporation.

Petrie, K., Chamberlain, K., \& Clarke, D. (1988). Psychological predictors of future suicidal behaviour in hospitalized suicide attempters. British Journal of Clinical Psychology, 27, 247-257.

Pokorny, A. D. (1993). Prediction of suicide in psychiatric patients: Report of a prospective study. In R. W. Maris, A. L. Berman, J. T. Maltsberger, \& R. I. Yufit (Eds.), Assessment and prediction of suicide. New York: Guilford Press.

Post, R. M. (1992). Transduction of psychosocial stress into the neurobiology of recurrent affective disorder. American Journal of Psychiatry, 149, 999-1010.

Rudd, M. D., Joiner, T. E., \& Rajab, M. H. (1996). Relationship among suicide ideators, attempters and multiple attempters in a young adult sample. Journal of Abnormal Psychology, 105, 541-550.

Salter, D., \& Platt, S. (1990). Suicidal intent, hopelessness and depression in a parasuicide population: The influence of social desirability and elapsed time. British Journal of Clinical Psychology, 29, 361371.

Schotte, D. E., Cools, J., \& Payvar, S. (1990). Problem-solving deficits in suicidal patients-trait vulnerability or state phenomenon. Journal of Consulting and Clinical Psychology, 58, 562-564.

Scott, J., House, R., Yates, M., \& Harrington, J. (1997). Individual risk factors for early repetition of deliberate self-harm. British Journal of Medical Psychology, 70, 387-393. 
Segal, Z. V., Gemar, M., \& Williams, S. (1999). Differential cognitive response to a mood challenge following successful cognitive therapy or pharmacotherapy for unipolar depression. Journal of Abnormal Psychology, 108, 3-10.

Segal, Z. V., \& Ingram, R. E. (1994) Mood priming and construct activation in tests of cognitive vulnerability to unipolar depression. Clinical Psychology Review, 14, 663-695.

Segal, Z. V., Kennedy, S., Gemar, M., Sagrati, S., Hood, K., \& Pedersen, R. (2003). Using mood induction to activate depression relapse vulnerability following cognitive or pharmacological treatment. Presented at the Society for Research in Psychopathology, Toronto, ON.

Segal, Z. V., Williams, J. M. G., Teasdale, J. D., \& Gemar, M. (1996). A cognitive science perspective on kindling and episode sensitization in recurrent affective disorder. Psychological Medicine, 26, 371380.

Segal, Z. V., Kennedy, S., Gemar, M., Hood, K., Pedersen, R., \& Buis, T. (2006). Cognitive reactivity to sad mood provocation and the prediction of depressive relapse. Archives of General Psychiatry, 63, $749-755$.

Sidley, G. L., Calam, R., Wells, A., Hughes, T., \& Whitaker, K. (1999). The prediction of parasuicide repetition in a high-risk group. British Journal of Clinical Psychology, 38, 375-386.

Szanto, K., Reynolds, C. F., Conwell, Y., Begley, A. E., \& Houck, P. (1998). High levels of hopelessness persist in geriatric patients with remitted depression and a history of attempted suicide. Journal of the American Geriatrics Society, 46, 1401-1406.

Taylor, L., \& Ingram, R. E. (1999). Cognitive reactivity and depressotypic information processing in children of depressed mothers. Journal of Abnormal Psychology, 108, 202-210.

Tversky, A., \& Kahneman, D. (1973). Availability: A heuristic for judging frequency and probability. Cognitive Psychology, 5, 207-232.

Van der Does, A. J. W. (2002a). Cognitive reactivity to a sad mood: structure and validity of a new measure. Behaviour Research \& Therapy, 40, 105-120.

Van der Does, A. J. W. (2002b). Manual of the Dutch version of the BDI-II [Handleiding bij de Nederlandse bewerking van de BDI-II]. San Antonio TX/Lisse NL: The Psychological Corporation/ Swets Test Publishers.

Van der Does, A. J. W. (2005). Thought suppression and cognitive vulnerability to depression. British Journal of Clinical Psychology, 44, 1-14.

Van der Does, A. J. W., Barnhofer, T., \& Williams, J. M. G. (2003). The Major Depression Questionnaire $(M D Q)$ www.dousa.nl/publications.

Van der Does, A. J. W., \& Williams, J. M. G. (2003). The Leiden Index of Depression Sensitivity (LEIDS). Revised version. Unpublished manuscript.

Wetzel, R. D., Margulies, T., Davis, R., \& Karam, E. (1980). Hopelessness, depression, and suicide intent. Journal of Clinical Psychiatry, 41, 159-160.

Weissman, A. N. (1979). The dysfunctional attitude scale. Philadelphia PA: University of Pennsylvania (Dissertation Abstracts International: 40, 1389B-1390B).

Williams, J. M. G., Barnhofer, T., Crane, C., \& Beck, A. T. (2005). Problem solving deteriorates following mood challenge in formerly depressed patients with a history of suicidal ideation. Journal of Abnormal Psychology, 114(3), 421-431.

Young, M. A., Fogg, L. F., Scheftner, W., Fawcett, J., Akiskal, H., \& Maser, J. (1996). Stable trait components of hopelessness: Baseline and sensitivity to depression. Journal of Abnormal Psychology, 105, 155-165. 